\title{
Bioengineering of archaeal flagella
}

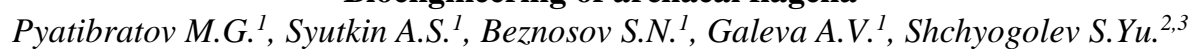

${ }^{1}$ Institute of Protein Research, Russian Academy of Sciences, Pushchino, Russia; ${ }^{2}$ Institute of Biochemistry and Physiology of Plants and Microorganisms, Russian Academy of Sciences, Saratov, Russia; ${ }^{3}$ Saratov National Research State University named after N.G. Chernyshevsky, Saratov, Russia

E-mail: bratov@vega.protres.ru

Key message. It was shown that the Haloferax volcanii flagella assembly system can accept alien flagellins and build functional recombinant flagella. The results can be used for targeted flagella modification to create multifunctional nanomaterials.

Keywords: halophilic archaea, flagellin, archaellin, gene expression, protein engineering

Bacterial and archaeal flagellins are remarkable for the presence of a highly conserved region (N- and C-termini) responsible for the polymerization properties and hypervariable regions in the central part of the molecule that forms the surface determinants of the flagellum. The presence of hypervariable sites makes flagella convenient objects of bioengineering, making it possible to create matrices for binding appropriate ligands. These variable flagellin parts can be modified by peptide inserts with high affinity for the selected ligand. As a result, an ordered arrangement of the ligand on the biopolymer matrix is achieved, and nanostructured materials with desired (conductive, magnetic, catalytic, sorption, etc.) properties can be obtained. Increased resistance to denaturing agents gives archaeal flagella (archaella) significant advantages over bacterial counterparts. Earlier we first constructed Halobacterium salinarum flagella modified with peptide inserts and synthesized nanomaterials with useful properties on their basis. We are currently working with the Haloferax volcanii strain, for which the most advanced haloarchaea system for genetic manipulation and protein expression has been developed. The aim of our study is to obtain $H f x$. volcanii strains producing recombinant flagella formed from modified flagellins and flagellins of other haloarchaea. Using heterologous expression, we obtained $H f x$. volcanii strains synthesizing functional flagella from flagellins of two distantly related archaeal species (Halorubrum lacusprofundi and Hbt. salinarum). To find out the roles of individual flagellins, we expressed the Hrr. lacusprofundi (flaB1, flaB2) and Hbt. salinarum (flgA1, flgA2) flagellin genes in the Hfx. volcanii strain in which the endogenous flagellin genes were deleted. Six Hfx. volcanii strains expressing the Hrr. lacusprofundi and Hbt. salinarum single flagellin genes or an operone from two genes produced functional filaments consisting of only one or both flagellins. All recombinant $H f x$. volcanii strains were motile, although significant differences were observed in motility efficiency. Recombinant filaments in structure and stability were similar to natural Hrr. lacusprofundi and Hbt. salinarum flagella. Electron microscopy showed that both FlaB1/FlaB2 and FlgA1/FlgA2 flagella look like typical supercoiled filaments, while the shape of one-component flagella is more variable. The heteropolymeric Hrr. lacusprofundi filaments have greater thermal stability and are more resistant to low salinity than single-component filaments. This shows that the thermal stability of these flagella depends on the presence of both flagellin types and indicates a close interaction between these subunits in the supramolecular structure. Functional spiral Hrr. lacusprofundi flagellum can consist of only one flagellin: FlaB2 or FlaB1, however, two different flagellins in combination provide additional stabilization of the flagellum structure, and, thus, adaptation to a wider range of external conditions. In the case of recombinant Hbt. salinarum filaments FlgA1/FlgA2, we did not observe significant differences in their thermal stability in comparison with singlecomponent recombinant filaments. Previously, the experiments on Hbt. salinarum strains with deleted flagellin genes demonstrated that filaments consisting only of FlgA1 (FlgA2) lose their functionality. Our results may indicate the influence of the genomic context, post-translational modifications, and other features of the host cell on the functional flagella assembly. Thus, we first showed that the $H f x$. volcanii flagella assembly system can accept alien flagellin genes and build functional recombinant flagella. By combining various flagellin types we can change the physical properties of synthesized filaments. Using 3D molecular modeling predictions and subsequent experimental verification we selected two optimal sites in the Hbt. salinarum archaellin for insertions of peptide sequences giving the archaella new properties for their use in creating nanomaterials with targeted properties. The results can clarify the general principles of the formation of macromolecular assemblies on cell surfaces and will be used in flagella bioengineering.

This work was supported by the Russian Foundation for Basic Research (grant No. 19-04-00613 A). 